\title{
A Novel Bridging Strategy Using Central Extracorporeal Membrane Oxygenation in Post-Myocardial Infarction Ventricular Septal Defect
}

\author{
Stephanie Nguyen ${ }^{1}$, Lucas Witer ${ }^{1}$, Yuji Kaku ${ }^{2}$, David Blitzer ${ }^{1}$, William Erwin ${ }^{1}$, and Koji \\ Takeda ${ }^{3}$ \\ ${ }^{1}$ New York-Presbyterian/Columbia University Medical Center \\ ${ }^{2}$ Columbia University Irving Medical Center \\ ${ }^{3}$ Columbia University Medical Center
}

December 21, 2020

\begin{abstract}
Ventricular septal defect (VSD) is a fatal mechanical complication of acute myocardial infarction (MI). The outcome of conventional surgery in post-MI VSD patients complicated by cardiogenic shock is extremely poor. We report two cases of a post-MI VSD stabilized with extracorporeal membrane oxygenation support followed by successful patch repair.
\end{abstract}

\section{Introduction}

With improvements in coronary revascularization, ventricular septal defect (VSD) has become a rare mechanical complication of myocardial infarction (MI). Mortality with conservative management remains over $94 \%$, emphasizing the need for surgery ${ }^{1}$. The most important determinant of survival is surgical timing, as there is a graded decline in mortality with time and myocardial healing. Operative mortality of VSD repair within 7 days of MI is $54.1 \%$ versus $18.4 \%$ if surgery is performed after 7 days. When repair is delayed by over 21 days, mortality is reduced to $10 \%^{2}$. Strategies that enable patient stabilization, allowing for optimal surgical timing are necessary. We report two cases of post-MI VSD managed with a novel approach using central extracorporeal membrane oxygenation ${ }^{3}$ followed by patch repair.

\section{Case 1}

A 53-year-old male presented to an outside hospital with a 3-hour history of chest pain. Electrocardiogram showed an inferior ST-elevation MI. Coronary angiography revealed 100\% mid-right coronary artery occlusion and a single drug-eluting stent (DES) was placed, as flow could not be restored distally. Given good collateral flow with preserved ejection fraction (EF 50\%), he was discharged after 2 days. He developed recurrent chest pain and was readmitted 3 days later in cardiogenic shock. Coronary angiography was unchanged with patent DES. Transthoracic echocardiography (TTE) revealed normal left ventricular function, severely depressed right ventricular function, hypokinetic inferolateral wall, and a $2 \mathrm{~cm}$ VSD at the inferior mid-septum with left-to-right shunting (Figure 1A). Given his worsening cardiogenic shock, femoral venoarterial extracorporeal membrane oxygenation (VA-ECMO) was initiated prior to transfer to our institution.

The patient arrived intubated with minimal pulsatility and a non-opening aortic valve with root stasis, requiring urgent conversion to central ECMO. Intraoperative transesophageal echocardiography (TEE) demonstrated a posterior VSD and aortic root thrombus (Figure 1B). The ascending aorta and right atrium were exposed through a right anterior mini-thoracotomy at the second intercostal space and the LV apex was 
exposed through a left anterior mini-thoracotomy at the fifth intercostal space (Figure 2). The ascending aorta and right atrium were cannulated with a 21-Fr arterial and 24-Fr venous cannula, respectively. Both cannulas were tunneled and exteriorized at the right upper chest. The LV apex was cannulated with a 24-Fr venous cannula and tunneled out to the left upper abdomen. LV apex and right atrial cannulas were connected with a Y-connector as inflows. All cannulas were connected to the CentriMag (Abbott Laboratories, Abbott Park, IL) and an oxygenator was spliced into the circuit. The femoral cannulas were removed. He was extubated on postoperative day 1 and participated in ambulatory rehabilitation. The patient remained on CentriMag support for 20 days with improving end-organ function before undergoing VSD closure.

After institution of cardiopulmonary bypass with bicaval cannulation, CentriMag flow was terminated and the existing cannulas removed. A double patch VSD closure was performed via the right ventricle ${ }^{4}$. The right ventriculotomy was made $1.5 \mathrm{~cm}$ from the mid-posterior descending artery and the VSD was identified, surrounded by firm fibrotic tissue (Figure 3). Two patches of bovine pericardium were tailored to the defect. The right ventriculotomy was closed and the patient came off bypass with inotropic support. TEE revealed EF 30-35\%, mildly decreased RV function, and no residual shunt. The postoperative course was uncomplicated and he was discharged home on postoperative day 12 with good functional status.

\section{Case 2}

A 73-year-old female presented with a 4-hour history of chest pain. Electrocardiogram showed an anterolateral ST-elevation MI. Coronary angiogram demonstrated complete occlusion of the mid-anterior descending artery and 60\% stenosis of the obtuse marginal branch. Thrombectomy and DES deployment were performed with excellent angiographic results. TTE revealed global biventricular hypokinesis (EF 30\%), apical septal akinesis, and a large mid-septal VSD with multiple left-to-right jets (Figure 1C). The patient rapidly developed cardiogenic shock with rising lactate $(3.0 \mathrm{mmol} / \mathrm{L})$ and underwent placement of an intra-aortic balloon pump (IABP) and femoral VA-ECMO. Three days later, TTE showed minimal aortic valve opening and root stasis, thus she was switched to central cannulation.

The patient underwent IABP removal and conversion to central ECMO with an LV apical vent (18-Fr aorta, 24-Fr RA, 22-Fr LV apex), as in Case 1. She was extubated on postoperative day 3 and participated in ambulatory rehabilitation. She remained hemodynamically stable with normalizing end-organ function for 22 days before undergoing definitive repair.

After establishment of cardiopulmonary bypass and regular cardiac arrest, CentriMag flow was terminated and the three existing cannulas were removed. Saphenous vein graft was used to bypass the obtuse marginal branch. A longitudinal right ventriculotomy was made $1 \mathrm{~cm}$ from the LAD and the $2 \mathrm{~cm}$ anterior VSD was identified in the mid-septum, surrounded by firm fibrotic tissue. We proceeded with a double patch VSD closure via the right ventricle, as in Case $1^{4}$. No residual VSD was seen on intraoperative TEE. The postoperative course was uncomplicated and the patient was extubated on postoperative day 4 . She was discharged to a rehabilitation facility on postoperative day 20 with improving functional status.

\section{Comment}

These cases illustrate employment of a unique central ECMO strategy to delay repair of post-MI VSD complicated by cardiogenic shock. Given the high mortality and low likelihood of successful repair in the immediate post-infarction period, both patients were stabilized with central ECMO for 3 weeks, granting time for recovery from cardiogenic shock and myocardial healing for successful patch closure.

Many mechanical circulatory support strategies have been used to stabilize patients before VSD repair ${ }^{5,6}$; however, each has its limitations. Percutaneous devices (i.e. IABP, Impella, TandemHeart) are readily placed and augment LV unloading and myocardial and peripheral tissue perfusion. However, such devices are limited by flow capability and inadequate support for biventricular failure, a common feature of post-MI VSD. Femoral VA-ECMO provides additional support but is limited by increased afterload and root stasis as seen in our cases. Furthermore, it limits ambulation, leading to progressive deconditioning. An axillaryjugular VA-ECMO configuration may also be used; however, flow is limited by arterial size and may lead to 
hyperperfusion of the arm and significant nerve injury if prolonged support is required.

Alternatively, a temporary external VAD may provide sufficient biventricular support in cardiogenic shock; however, insertion requires a sternotomy and cardiopulmonary bypass. Our approach addresses each of these limitations. Firstly, it facilitates biventricular unloading while providing full cardiopulmonary support with antegrade oxygenated flow. Additionally, it allows preservation of a virgin chest, with very minimal intrapericardial adhesions noted, for the planned VSD closure and permits ambulatory therapy prior to surgical repair.

VSD closure was performed using a double-patch repair through a right ventriculotomy. In this novel technique, two patches are anchored to the VSD margin using interrupted horizontal mattress sutures placed transeptally or transmurally from the LV (via the VSD) to the RV cavity or LV wall, creating a "sandwich" closure. There are many advantages to this approach, including preservation of contractile function by avoiding a left ventriculotomy and reproducibility for anterior and posterior defects.

In conclusion, our approach is a feasible bridge strategy in the management of post-MI VSD with cardiogenic shock. Further experience with this modality is required.

\section{Author Contributions}

Stephanie N. Nguyen, MD: concept/design, drafting article, critical revision of article, Lucas J. Witer, MD: critical revision of article, approval of article

Yuji Kaku, MD: critical revision of article,

David Blitzer, MD: drafting article, critical revision of article

William C. Erwin, MD: critical revision of article

Koji Takeda, MD: concept/design, drafting article, critical revision of article, approval of article

\section{References}

Crenshaw BS, Granger CB, Birnbaum Y, et al. Risk Factors, Angiographic Patterns, and Outcomes in Patients With Ventricular Septal Defect Complicating Acute Myocardial Infarction. Circulation . 2000;101(1):27-32.

Arnaoutakis GJ, Zhao Y, George TJ, et al. Surgical Repair of Ventricular Septal Defect After Myocardial Infarction: Outcomes From The Society of Thoracic Surgeons National Database. The Annals of Thoracic Surgery . 2012;94(2):436-444.

1. Takeda K, Garan AR, Topkara VK, et al. Novel minimally invasive surgical approach using an external ventricular assist device and extracorporeal membrane oxygenation in refractory cardiogenic shock.European Journal of Cardio-Thoracic Surgery . June 2016.

2. Asai T, Hosoba S, Suzuki T, et al. Postinfarction ventricular septal defect: right ventricular approachthe extended "Sandwich" patch.Seminars in Thoracic and Cardiovascular Surgery . 2012;24(1):59-62.

3. Patanè F, Grassi R, Zucchetti MC, et al. The use of Impella Recover in the treatment of post-infarction ventricular septal defect: A new case report. International Journal of Cardiology . 2010;144(2):313-315.

4. Gregoric ID, Kar B, Mesar T, et al. Perioperative Use of TandemHeart Percutaneous Ventricular Assist Device in Surgical Repair of Postinfarction Ventricular Septal Defect. ASAIO Journal . 2014;60(5):529532. 


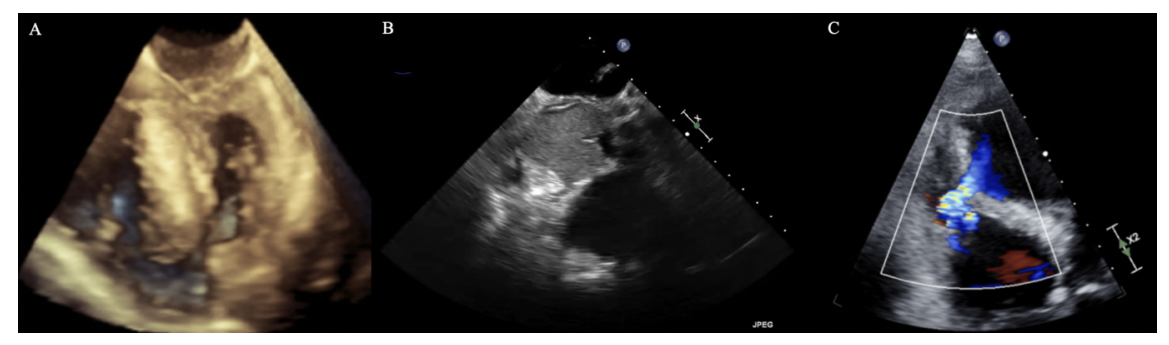

Figure 1. A) Intraoperative TEE 3D reconstruction demonstrating posterior apical VSD. B) Intraoperative TEE demonstrating aortic root thrombus C) Anterior VSD with left-to-right shunting seen on preoperative TTE

$T E E=$ transesophageal echocardiography $; T T E=$ transthoracic echocardiography $;$ VSD = ventricular septal defect 


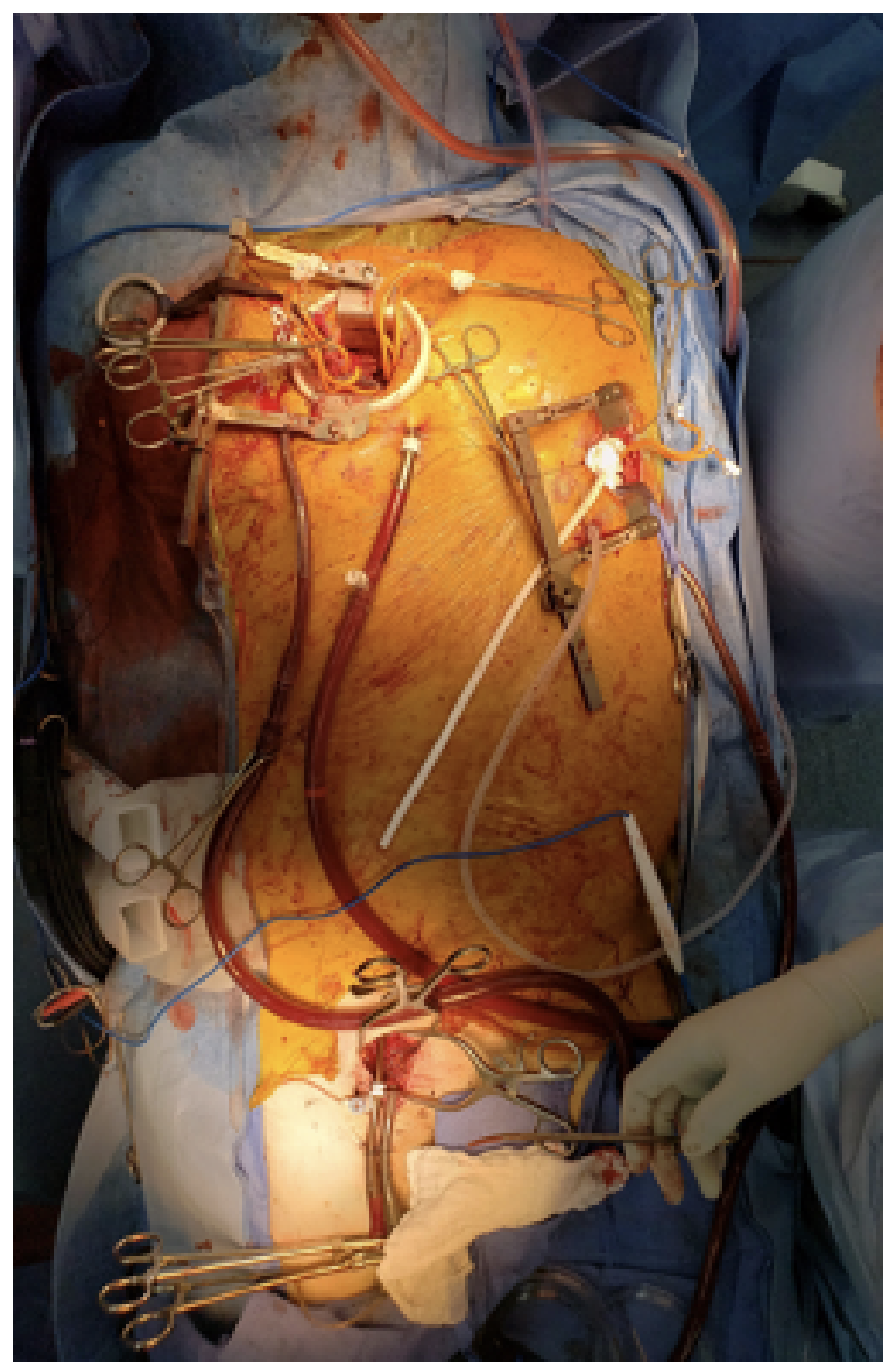

Figure 2. Central VA-ECMO with LV vent configuration. Inflow = right atrium and left ventricular apex; outflow $=$ ascending aorta. Femoral cannulas are clamped for decannulation.

$V A-E C M O=$ venoarterial extracorporeal membrane oxygenation 


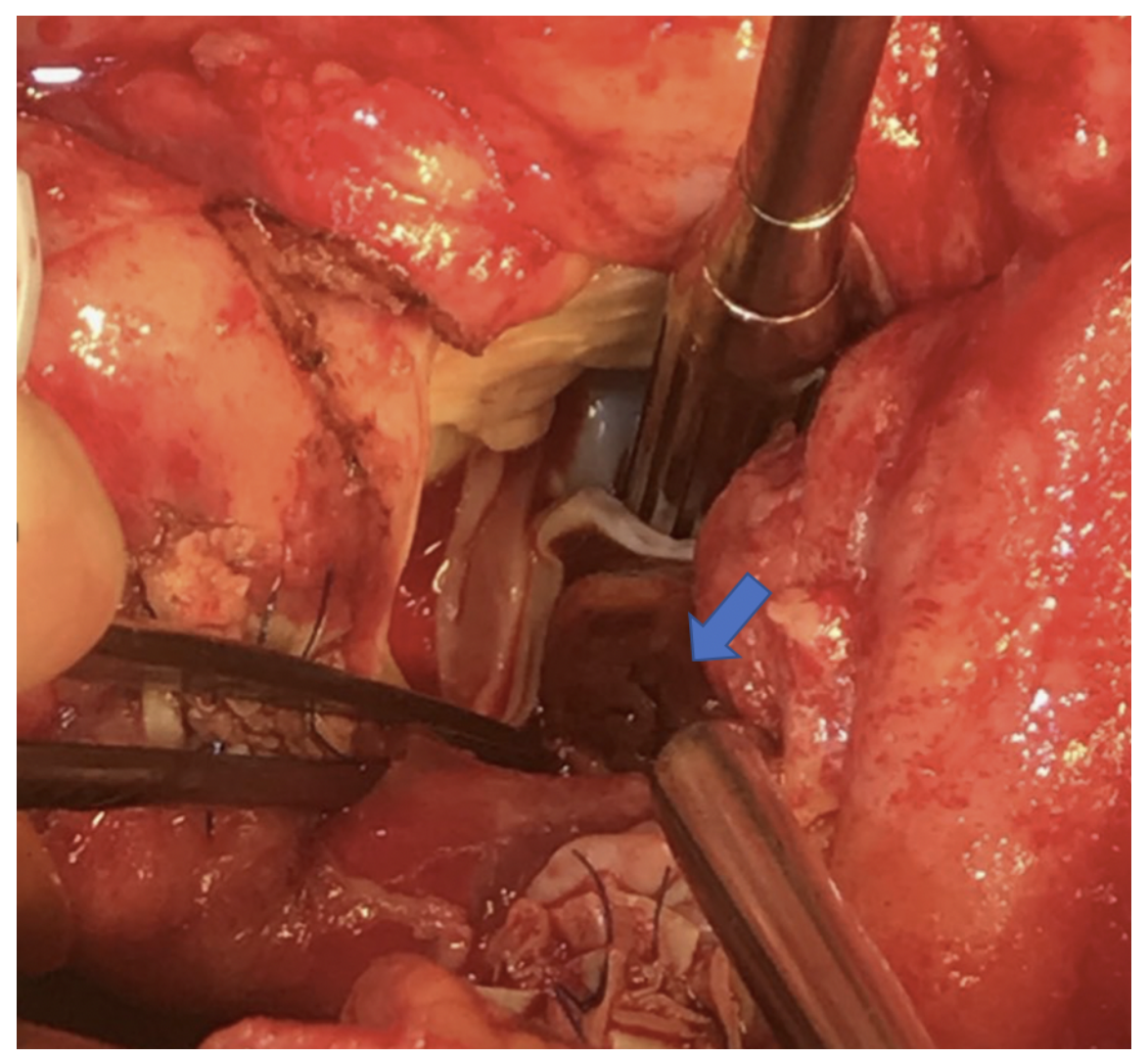

S

Figure 3. Posterior ventricular septal defect (blue arrow) visualized through an inferior right ventriculotomy.

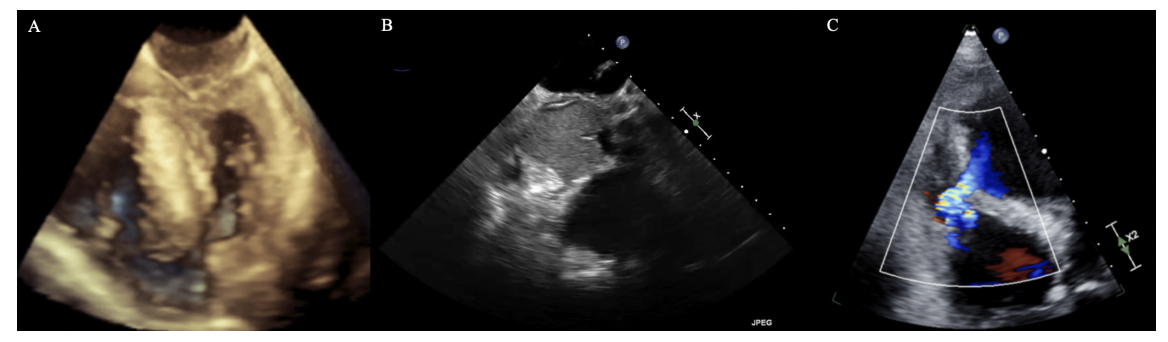




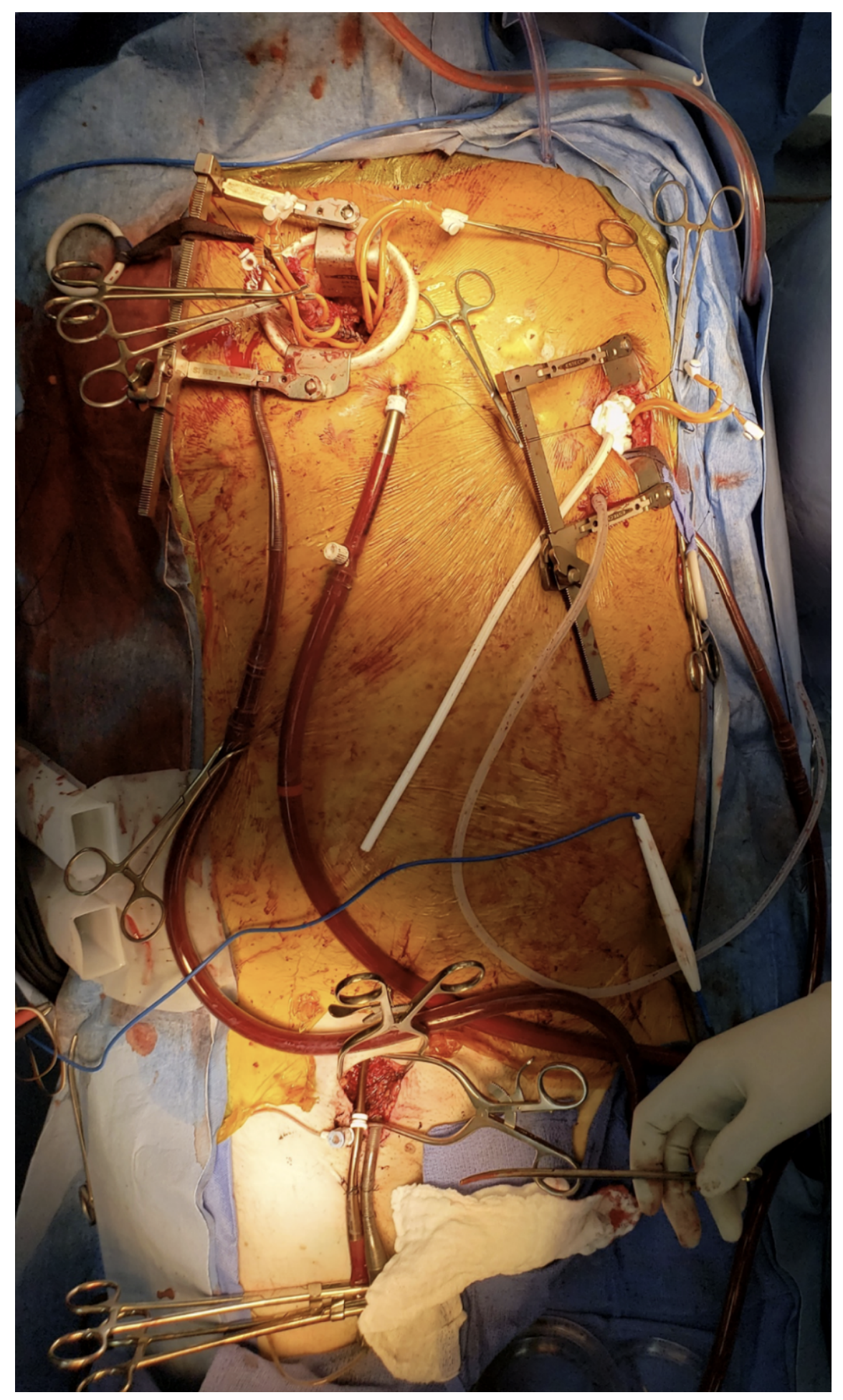




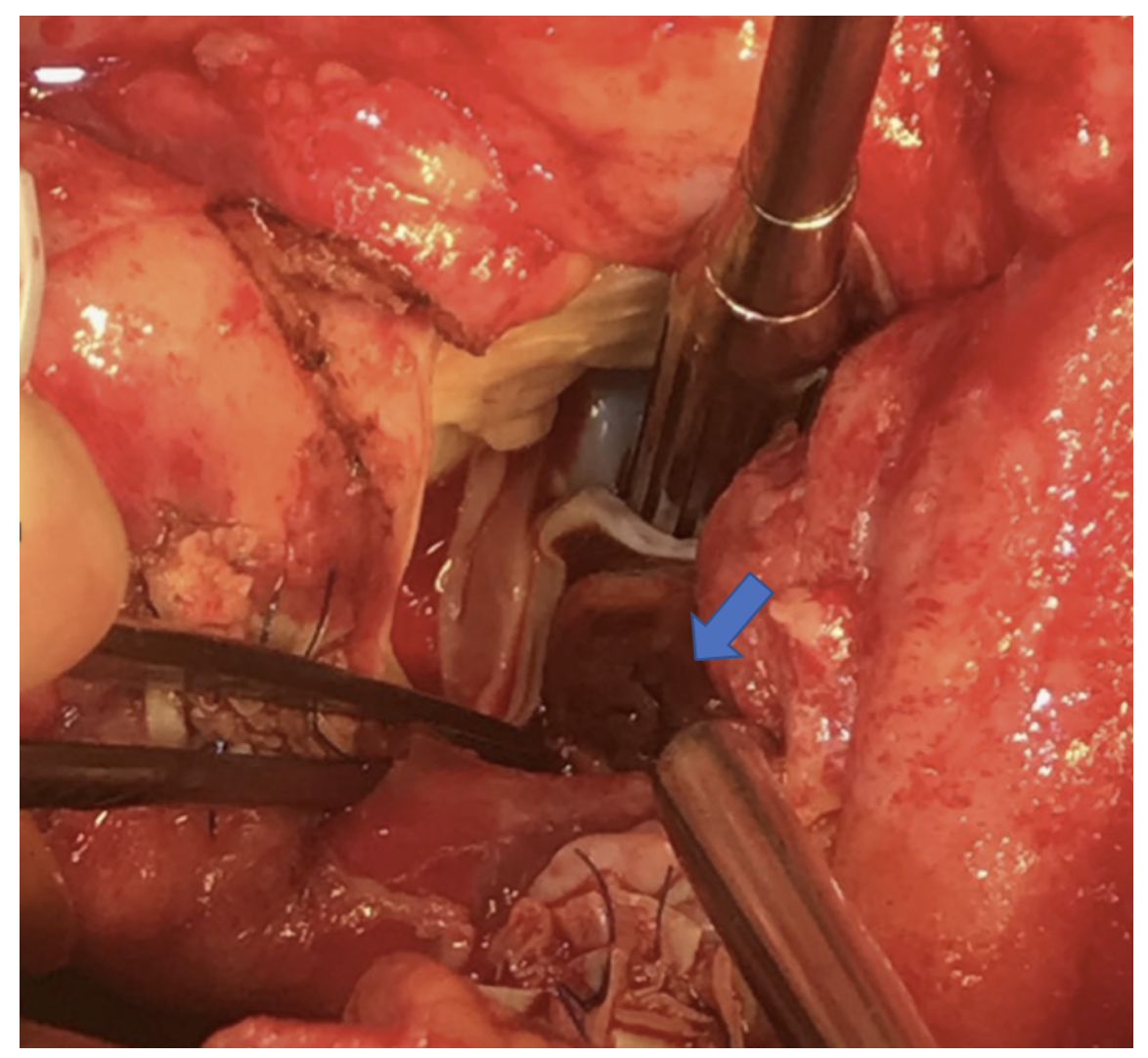

